\title{
The Role of Osmotic Effects in Haloadaptation of Vibrio costicola
}

\author{
By R. ADAMS, ${ }^{1}$ J. BYGRAVES, ${ }^{1}$ M. KOGUT ${ }^{1,2}$ AND N. J. RUSSELL ${ }^{1 *}$ \\ ${ }^{1}$ Department of Biochemistry, University College, PO Box 78, Cardiff CFI IXL,UK \\ 2 Department of Biochemistry, King's College London (KQC), Campden Hill Road, Kensington, \\ London W8 7AH, UK
}

(Received 1 December 1986; revised 19 February 1987)

\begin{abstract}
Growth rates of Vibrio costicola showed a broad optimum between 0.8 and $1.5 \mathrm{M}-\mathrm{NaCl}$, and there was no growth above $3.3 \mathrm{M}-\mathrm{NaCl}$ in a peptone-based medium. The minimum requirement of $0.5 \mathrm{M}-\mathrm{NaCl}$ for growth in $\mathrm{NaCl}$ alone was reduced to $0.3 \mathrm{M}-\mathrm{NaCl}$ when the total solute concentration was raised to 0.5 to $1.0 \mathrm{M}$ equivalent with sucrose or glycerol. Compared with equivalent $\mathrm{NaCl}$ concentrations, higher concentrations of sucrose were more inhibitory to growth, whereas glycerol had less effect. Increasing the medium $\mathrm{NaCl}$ concentration suddenly by 2- or 3-fold with either a constant starting, or final, salt concentration showed that, after the shift-up, the lag in growth, the rate of growth, and the inhibition of phospholipid synthesis depended both on the final $\mathrm{NaCl}$ concentration and the magnitude of the shift in salinity. The time-courses of phospholipid synthesis following a 2- or 3-fold shift-up in $\mathrm{NaCl}$ or sucrose media were very similar and exhibited a relative increase in phosphatidylglycerol synthesis over that of phosphatidylethanolamine. This 'switch-over' was not seen following shift-up in glycerol media when there was also a stimulation, rather than inhibition, of phospholipid synthesis. It is concluded that during phenotypic haloadaptation of $V$. costicola, osmotic effects play a significant part in the sensing of and response to raised external salinity.
\end{abstract}

\section{INTRODUCTION}

Moderately halophilic bacteria provide a suitable system for studying adaptive changes in membrane structure and function, because the phospholipid composition of several species, including Vibrio costicola, depends on the salinity of the growth medium (Hanna et al., 1984; Miller, 1985). When growing cultures of $V$. costicola are subjected to sudden shifts in salt concentration, the phospholipid composition alters during the period immediately after the shift, when growth slows or stops (Kogut \& Russell, 1984). This change is brought about by changes in biosynthetic rates of individual phospholipids, rather than degradation of membrane phospholipids (Russell et al., 1985). After a shift-up from 1 to $3 \mathrm{M}-\mathrm{NaCl}$ there is an immediate, drastic reduction in phosphatidylethanolamine (PE) synthesis and a smaller decrease in phosphatidylglycerol (PG) synthesis; these inhibitions are immediately reversible if the external salt concentration is restored to the original, i.e. pre-shift, value (Russell et al., 1985). The initial inhibition of phospholipid synthesis after such a shift-up is followed by a rapid increase in PG synthesis above the rate of PE synthesis and a slower recovery of the latter. Both synthetic rates eventually reach constant values when the culture attains steady growth at a rate appropriate to the new salt concentration.

Two questions arise following these observations. (1) What do the bacteria sense when confronted by a sudden change in external salt concentration - the extent of change or the actual concentration of salt in the new medium (i.e. is there a kind of threshold)? (2) What part is

Abbreviations: CL, cardiolipin (diphosphatidylglycerol); PE, phosphatidylethanolamine; PG, phosphatidylglycerol. 
played by the osmotic effect of high external salinity, rather than specific salt effects, in the process of haloadaptation by $V$. costicola?

To answer the first question, we have compared the adaptive behaviour, namely growth and phospholipid metabolism, of $V$. costicola after shifts of equal magnitude but with different final salt concentrations, and shifts of different magnitude but with the same final salt concentration. To answer the second question, we have compared the adaptive behaviour of $V$. costicola after shifts in media containing uncharged solutes with its behaviour after shifts in equivalent concentrations of $\mathrm{NaCl}$.

Four aspects of the haloadaptive response have been investigated, namely: (a) the time-course of growth, i.e. the presence and extent of a lag period and the attainment of the final growth rate, characteristic of the new salt concentration; $(b)$ the phospholipid composition, i.e. the ratio of zwitterionic to anionic phospholipids (PE/PG); $(c)$ the reversible, immediate inhibition in the rates of synthesis of $P E$ and $P G$; and $(d)$ the time-course of rates of $P G$ and $P E$ synthesis, following the initial inhibition. Some preliminary results of these investigations have already been published (Russell et al., 1986).

\section{METHODS}

Organism and cultural conditions. The methods of maintaining and culturing Vibrio costicola (NRCC 3700) in media of different salt concentrations, and for changing suddenly (shifting) the salt concentration are described by Russell et al. (1985). The same methods were used for media containing sucrose or glycerol. Sucrose and glycerol media contained 0.5 or $1.0 \mathrm{M}-\mathrm{NaCl}$ as indicated plus sufficient sucrose or glycerol to give the required 'equivalent molarity' (i.e. a final concentration of solutes giving the same osmotic effect as would $\mathrm{NaCl}$ alone), using the conversion tables in Wolf et al. (1981). For simplicity, such media are referred to as 'sucrose' or 'glycerol' media, although they also contained salt. Sucrose and glycerol were used as the non-ionic solutes, the former as a nonpenetrating and the latter as a readily penetrating one. Penetration was assessed by the ability of the solute to cause plasmolysis, measured using the light-scattering method of Schnaiderman \& Avi-Dor (1982). $\mathrm{NaCl}$ is relatively non-penetrating and sudden shifts to high concentrations of $\mathrm{NaCl}$ produce plasmolysis in $V$. costicola, at least for a time (Kushner et al., 1983; M. Kogut, unpublished results).

Cultures in Erlenmeyer flasks containing $20 \%$ volume of medium were grown at $30^{\circ} \mathrm{C}$ in an orbital incubator. Growth was monitored by $\mathrm{OD}_{500}$ measurements (Kogut \& Russell, 1984). The relationship between $\mathrm{OD}_{500}$ and bacterial dry wt was the same for each medium after correcting $\mathrm{OD}_{500}$ values for the light-scattering effects of sucrose and glycerol. In some experiments tube assays of growth yield were done: tubes containing 5 or $10 \mathrm{ml}$ protease peptone/tryptone (Difco) medium (Russell et al., 1985) were inoculated with one drop of an exponentially growing culture $\left(\mathrm{OD}_{500}=0.5\right.$ to 0.8$)$ and incubated at $30^{\circ} \mathrm{C}$ with occasional shaking.

Measurement of phospholipid composition. Phospholipids were extracted from cell pellets using the method of Bligh and Dyer as detailed by Kates (1972). Individual phospholipids were separated by TLC and assayed by phosphorus analysis as described previously (Kogut \& Russell, 1984; Russell et al., 1985).

Radioisotopes. Sodium $\left[1-^{14} \mathrm{C}\right]$ acetate $\left(1.90 \mathrm{GBq} \mathrm{mmol}^{-1}\right)$ was purchased from Amersham.

Measurement of phospholipid synthesis. The rate of synthesis of phospholipids was measured by the incorporation of $\left[1{ }^{-14} \mathrm{C}\right]$ acetate as described by Russell $\mathrm{et}$ al. (1985), with the following minor differences: phospholipids were separated by TLC on commercial silica gel plates (Merck); radioactivity was measured by liquid scintillation counting of samples in $5 \mathrm{ml}$ Optiphase-X scintillation cocktail (LKB). The kinetics of $\left[1-^{-14} \mathrm{C}\right]$ acetate incorporation in phospholipids of cells growing in non-ionic media were not significantly different to those growing in $\mathrm{NaCl}$ media.

\section{RESULTS}

\section{Growth after shifts-up in $\mathrm{NaCl}$ media}

To determine whether $V$. costicola can sense solute concentration per se, or whether it responds to the increase in salt concentrations, we did two types of shift experiments. In the first type the starting $\mathrm{NaCl}$ concentration was the same but the final concentration was different; in the second type the starting concentration was varied but the final (higher) $\mathrm{NaCl}$ concentration was the same. In both types of experiment the $\mathrm{NaCl}$ concentration was increased by 2- or 3-fold. In Fig. 1 the final growth rates of the shifted-up cultures expressed as a percentage of that of the controls (i.e. pre-shift) are plotted against the $\mathrm{NaCl}$ concentrations in the shift medium. For any 


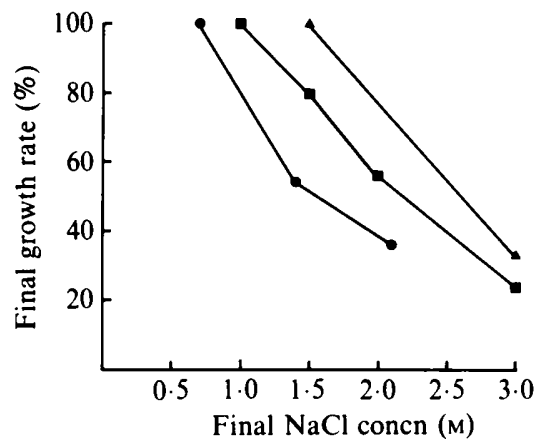

Fig. 1. Effect of shift-up in $\mathrm{NaCl}$ concentration on final growth rate of $\boldsymbol{V}$. costicola. Cultures growing in $0.7 \mathrm{M}(\mathrm{O}), 1.0 \mathrm{M}(\square)$ or $1.5 \mathrm{M}(\triangle) \mathrm{NaCl}$ were shifted-up to the $\mathrm{NaCl}$ concentrations indicated. The growth rates of shifted-up cultures are expressed as a percentage of that of the culture in the pre-shift medium.

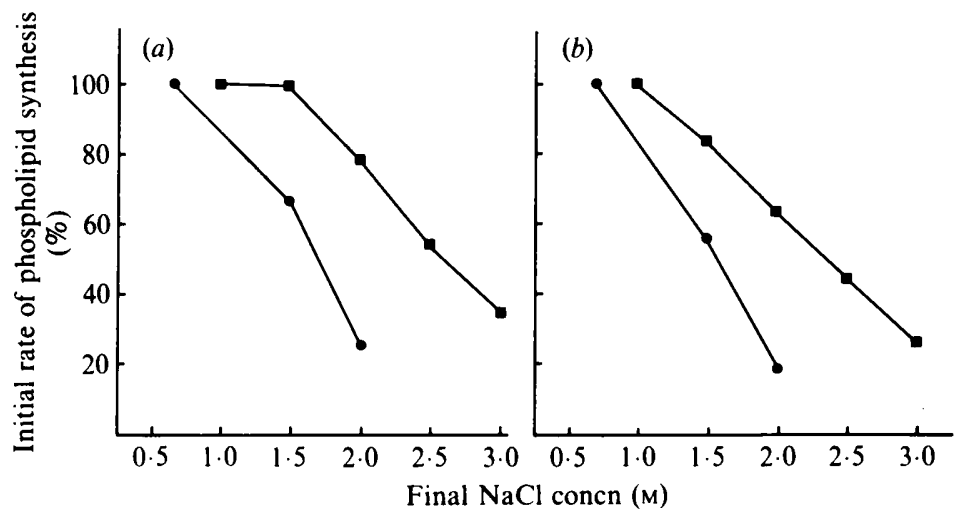

Fig. 2. Effect of shift-up in $\mathrm{NaCl}$ concentration on immediate phospholipid synthesis rates in $V$. costicola. Cultures growing in $0.7 \mathrm{M}(O)$ or $1.0 \mathrm{M}(\square) \mathrm{NaCl}$ were shifted-up to the $\mathrm{NaCl}$ concentrations indicated. Phospholipid synthesis was measured over a $15 \mathrm{~min}$ period, starting $5 \mathrm{~min}$ after the shift-up; the rates of phosphatidylglycerol $(a)$ and phosphatidylethanolamine $(b)$ synthesis are expressed as a percentage of the rates of pre-shift controls.

final $\mathrm{NaCl}$ concentration the extent of reduction in growth rate from the control depended on the difference between the pre-shift and final $\mathrm{NaCl}$ concentration, i.e. on the magnitude of the shift, and not on the actual concentration of $\mathrm{NaCl}$ in the shift medium.

We also observed that following certain shifts-up there was a lag period, the length of which was not always easily measured because growth did not always cease completely after a shift-up. In repeated experiments there were variations which seemed to depend partly on the extent of the shift and final $\mathrm{NaCl}$ concentration, partly on the rate of growth in the original $\mathrm{NaCl}$ concentration (i.e. pre-shift), and partly on the degree of 'stress' experienced during the manipulations. We were at pains to standardize the experimental protocol; under otherwise identical conditions lag times were shortest when centrifugation time and speeds were minimal and the temperature kept close to $25^{\circ} \mathrm{C}$, and when bacterial pellets were resuspended gently by swirling or using a rotary mixer. Notwithstanding experimental variations, for a 3-fold increase in $\mathrm{NaCl}$ concentration, a lag period was always observed when the final concentration was $>2 \mathrm{M}$ (data not shown). For a 2-fold increase in $\mathrm{NaCl}$ concentration, on the other hand, a lag was not always observed, unless the final concentration was $3 \mathrm{M}-\mathrm{NaCl}$ (data not shown).

\section{Phospholipid synthesis after increases in salt concentration}

The extent of immediate inhibition of phospholipid synthesis (as a percentage of control rates of phospholipid synthesis) is plotted against the $\mathrm{NaCl}$ concentration in the shift medium in Fig. 2. The initial inhibition of phospholipid synthesis for a given concentration of $\mathrm{NaCl}$ in the shift- 

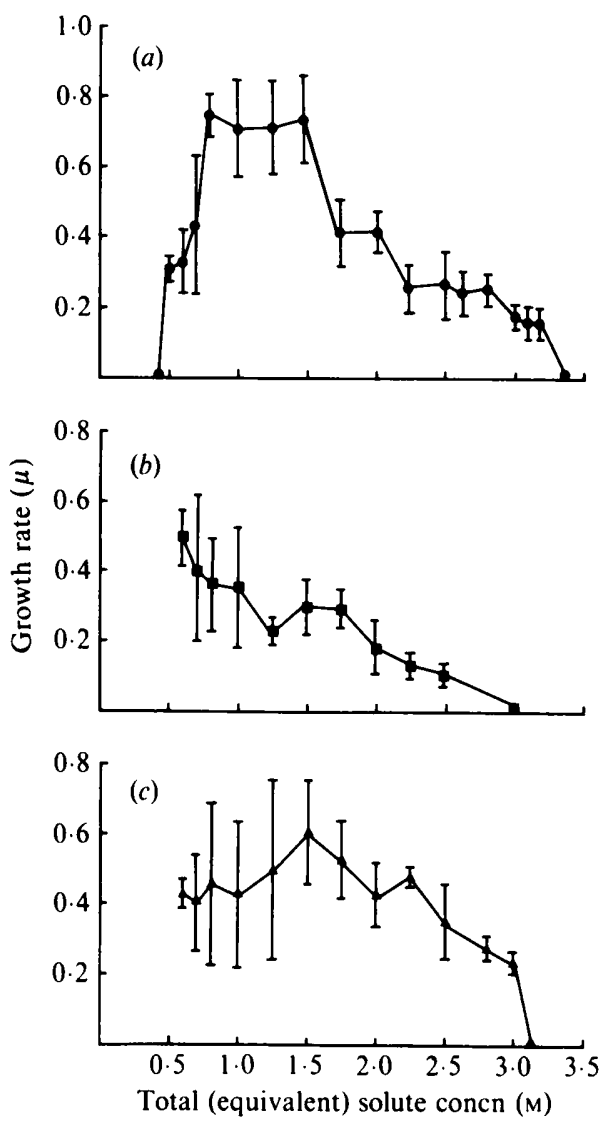

Fig. 3. Growth rates of $V$. costicola growing in media containing $\mathrm{NaCl}(a)$, sucrose $(b)$ or glycerol $(c)$. In $(b)$ and $(c)$, at total equivalent solute concentrations up to $1.25 \mathrm{M}$, the $\mathrm{NaCl}$ concentration was $0.5 \mathrm{M}$; for greater equivalent solute concentrations $1 \mathrm{M}-\mathrm{NaCl}$ was used. Bars represent $\pm \mathrm{SD}(n=3$ to 18$)$.

Table 1. Growth rates of $\mathrm{V}$. costicola in media containing different amounts of $\mathrm{NaCl}$ with sucrose or glycerol

Additional solute

\begin{tabular}{|c|c|c|c|c|c|c|}
\hline $\begin{array}{c}\mathrm{NaCl} \\
\text { concn (M) }\end{array}$ & $\begin{array}{l}\text { Growth } \\
\text { rate }(\mu)\end{array}$ & $\begin{array}{c}\text { Sucrose } \\
\text { concn }(M) \dagger\end{array}$ & $\begin{array}{l}\text { Growth } \\
\text { rate }(\mu)\end{array}$ & $\begin{array}{c}\text { Glycerol } \\
\text { concn }(\mathbf{M}) \dagger\end{array}$ & $\begin{array}{l}\text { Growth } \\
\text { rate }(\mu)\end{array}$ & $\begin{array}{l}\text { Total solute } \\
\text { concn }(\mathrm{M}) \dagger\end{array}$ \\
\hline $0 \cdot 2^{*}$ & & 0.8 & 0 & & & 1.0 \\
\hline $0.3^{*}$ & & 0.7 & $0 \cdot 16$ & 0.7 & 0.12 & 1.0 \\
\hline $0.4^{*}$ & & 0.6 & 0.24 & 0.6 & 0.13 & 1.0 \\
\hline 0.5 & 0.31 & & & & & 0.5 \\
\hline 0.5 & & $0 \cdot 1$ & 0.56 & & & 0.6 \\
\hline 0.5 & & 0.2 & 0.58 & & & 0.7 \\
\hline 0.5 & & 0.3 & 0.51 & & & 0.8 \\
\hline 0.5 & & 0.5 & 0.71 & 0.5 & 0.46 & 1.0 \\
\hline 0.6 & 0.32 & & & & & \\
\hline 0.7 & 0.42 & & & & & \\
\hline 0.8 & 0.74 & & & & & \\
\hline $1 \cdot 0$ & 0.71 & & & & & \\
\hline
\end{tabular}

* No growth occurred in $0 \cdot 2$ to $0.4 \mathrm{M}-\mathrm{NaCl}$ media without additional solute. $\dagger$ Equivalent solute concentration. 
medium depended on the magnitude of the shift. Thus a shift-up from 0.7 to $2 \mathrm{M}-\mathrm{NaCl}$ gave very much more inhibition than from I to $2 \mathrm{M}-\mathrm{NaCl}$ and was similar to that seen after a shift-up from 1 to $3 \mathrm{M}-\mathrm{NaCl}$ (Fig. 2). The inhibition of PE synthesis was generally greater than that of PG synthesis.

\section{Growth in different solutes}

The growth rates of cultures growing in media containing a range of equivalent osmolarities of $\mathrm{NaCl}$, sucrose or glycerol are shown in Fig. 3. Since the organism has an apparent minimum requirement of $0.5 \mathrm{M}-\mathrm{NaCl}, \mathrm{NaCl}$ was present in each medium - viz. at $0.5 \mathrm{M}$ when the total equivalent concentration was $₹ 1.25 \mathrm{M}$, and at $1.0 \mathrm{M}$ when the total equivalent concentration was $>1.5 \mathrm{M}$. Experiments with $0.5 \mathrm{M}-\mathrm{NaCl}$ at a total concentration of $1.5 \mathrm{M}$, or with $1.0 \mathrm{M}-\mathrm{NaCl}$ at a total of $1.25 \mathrm{M}$, showed that the change in proportion of $\mathrm{NaCl}$ at these intermediate total equivalent concentrations did not affect growth rates.

The large standard deviations in growth rates were caused by variation between one experimental set of cultures and another. Within any set of cultures the same relationship between growth rates and $\mathrm{NaCl}$ concentration was observed, although the absolute values of growth rates varied between experiments. In order to check these data, an alternative experimental approach was adopted in which growth yield was measured as the optical density in a series of tube cultures containing the range of salt concentrations (data not shown). Under these conditions growth yield (and approximate rate) was much less than in fully aerated, shaken flask cultures, but the same qualitative results were obtained using each experimental system.

No growth occurred below $0.5 \mathrm{M}-\mathrm{NaCl}$ (Fig. $3 a$ ). However, growth rates rose steeply with higher salt concentrations up to approximately $0.8 \mathrm{M}$. There was a broad plateau in growth rates between approximately 0.8 and $1.5 \mathrm{M}-\mathrm{NaCl}$, followed by a shallow decline to $3.0 \mathrm{M}-\mathrm{NaCl}$. The upper growth limit was approximately $3 \cdot 3 \mathrm{M}-\mathrm{NaCl}$, but this was particularly difficult to evaluate because of the problem of measuring low culture optical densities that were changing very slowly.

In sucrose media (Fig. $3 b$ ), with 0.5 or $1.0 \mathrm{M}-\mathrm{NaCl}$ present as indicated, there was no true optimum sucrose concentration. Instead, a gradual decline in growth rate was observed as the solute concentration was increased above $0.6 \mathrm{M}$, until no growth occurred at $3.0 \mathrm{M}$ total equivalent solute.

In glycerol media (Fig. 3c), on the other hand, the response was broadly similar to that with $\mathrm{NaCl}$ alone, but the optimum was less well defined.

\section{Minimum requirement for salt}

In order to determine whether the apparent minimum requirement for $0.5 \mathrm{M}-\mathrm{NaCl}$ was an absolute one, or whether some of the salt could be replaced by a non-ionic solute, the growth rates of $V$. costicola were measured in media containing $₹ 0.5 \mathrm{M}-\mathrm{NaCl}$ and made up to total equivalent solute concentrations of $0.6-1.0 \mathrm{M}$ with sucrose or glycerol (Table 1). Non-ionic solutes could replace part of the $\mathrm{NaCl}$ requirement, and they lowered the minimum requirement to $0.3 \mathrm{M}-\mathrm{NaCl}$ when the total solute equivalent concentration was made up to $1.0 \mathrm{M}$ with sucrose or glycerol. Table 1 also shows that at intermediate concentrations (i.e. 0.6-1.0 M) some combinations of $0.5 \mathrm{M}-\mathrm{NaCl}$ with varying concentrations of sucrose gave higher growth rates than did the equivalent concentration of $\mathrm{NaCl}$ alone. These studies were complemented by a series of tube assays for growth yields (data not shown), which also gave a minimum requirement of $0.3 \mathrm{M}-\mathrm{NaCl}$ in sucrose or glycerol media, and showed that the upper growth limit was $2.7 \mathrm{M}$ sucrose or 3.2 M-glycerol. Growth did not occur in media containing $1 \mathrm{M}$-sucrose or glycerol, but no $\mathrm{NaCl}$.

\section{Effects of shifts-up in different solutes on the immediate rates of phospholipid synthesis}

Cultures were shifted-up from $1 \mathrm{M}-\mathrm{NaCl}$ into media containing various final concentrations of $\mathrm{NaCl}$, sucrose or glycerol; all sucrose and glycerol media contained $1 \mathrm{M}-\mathrm{NaC}$. The effects of 

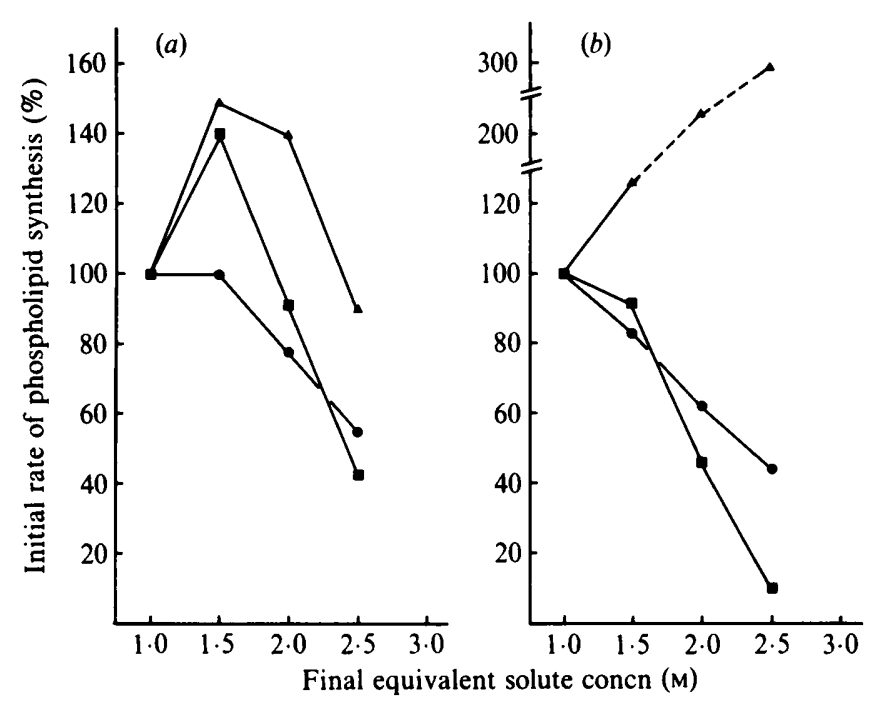

Fig. 4. Effect of shift-up in different solutes on immediate phospholipid synthesis rates in $V$. costicola. Cultures growing in $1 \mathrm{M}-\mathrm{NaCl}$ were shifted-up in media containing final (equivalent) concentrations of $\mathrm{NaCl}(\boldsymbol{O})$, sucrose ( $)$ or glycerol $(\boldsymbol{W})$ as indicated. Phospholipid synthesis was measured over a $15 \mathrm{~min}$ period, starting 5 min after the shift-up, and the rates of phosphatidylglycerol $(a)$ and phosphatidylethanolamine $(b)$ synthesis are expressed as a percentage of the rates in pre-shift $1 \mathrm{M}-\mathrm{NaCl}$ controls.

such shifts on the initial (i.e. 5 min after shift-up) rates of PG and PE synthesis are shown in Fig. 4. There was immediate inhibition of PE synthesis following shifts-up in $>1.5 \mathrm{M}-\mathrm{NaCl}$ media, and of $\mathrm{PG}$ synthesis in $>2.0 \mathrm{M}-\mathrm{NaCl}$ media. The inhibition of $\mathrm{PE}$ synthesis was always greater than that of PG synthesis.

The effects of shifts-up in sucrose media were qualitatively similar to those observed in $\mathrm{NaCl}$ media (Fig. 4). However, at the lowest concentration ( $1.5 \mathrm{M})$ there was no significant inhibition of PE synthesis and a marked stimulation of PG synthesis, whilst at the highest concentration $(2.5 \mathrm{M})$ the inhibition of PE synthesis was more severe than at an equivalent concentration of $\mathrm{NaCl}$; the effects on PG synthesis were similar at $2.5 \mathrm{M}$ for sucrose and $\mathrm{NaCl}$ media.

Shifts-up in glycerol media, on the other hand, gave a completely different result (Fig. 4). There was a marked stimulation of PE synthesis at all concentrations of glycerol and of PG synthesis in $<2.5 \mathrm{M}$-glycerol media.

\section{Phospholipid composition of $V$. costicola grown in different solutes}

When cultures of $V$. costicola were grown in media containing increasing amounts of sucrose, there was an increase in the relative proportion of anionic phospholipids, i.e. PG and cardiolipin (CL). The decreases in $\mathrm{PE} / \mathrm{PG}$ and $\mathrm{PE} / \mathrm{PG}+\mathrm{CL}$ ratios were very similar to those observed when medium osmolarity was raised with $\mathrm{NaCl}$ alone (data not shown). In media containing increasing amounts of glycerol, these ratios also decreased, but to a lesser extent, especially when the increase in $\mathrm{CL}$ was considered (data not shown). There was no significant change in the proportion of $\mathrm{CL}$ relative to $\mathrm{PG}$ in media containing increasing amounts of $\mathrm{NaCl}$ or sucrose.

\section{Effects of shifts-up in different solutes on the time-course of phospholipid synthesis}

The time-courses of phospholipid synthesis following shifts-up from $1 \mathrm{M}-\mathrm{NaCl}$ to 2 and $3 \mathrm{M}$ equivalent concentrations of $\mathrm{NaCl}$, sucrose or glycerol are shown in Fig. 5. The changes in rates of $\mathrm{PG}$ and $\mathrm{PE}$ synthesis after shifts-up in $\mathrm{NaCl}$ and sucrose media were very similar (cf. Fig. $5 a, b, c, d)$. The initial inhibition of phospholipid synthesis, which was more severe for PE than for PG (Fig. 4), was followed by a recovery in rates of synthesis with a much steeper increase in PG synthesis than PE synthesis (Fig. $5 a, b, c, d$ ). The rates of PG synthesis remained higher than PE synthesis throughout the time-course (Fig. $5 a, b, c, d$ ). 

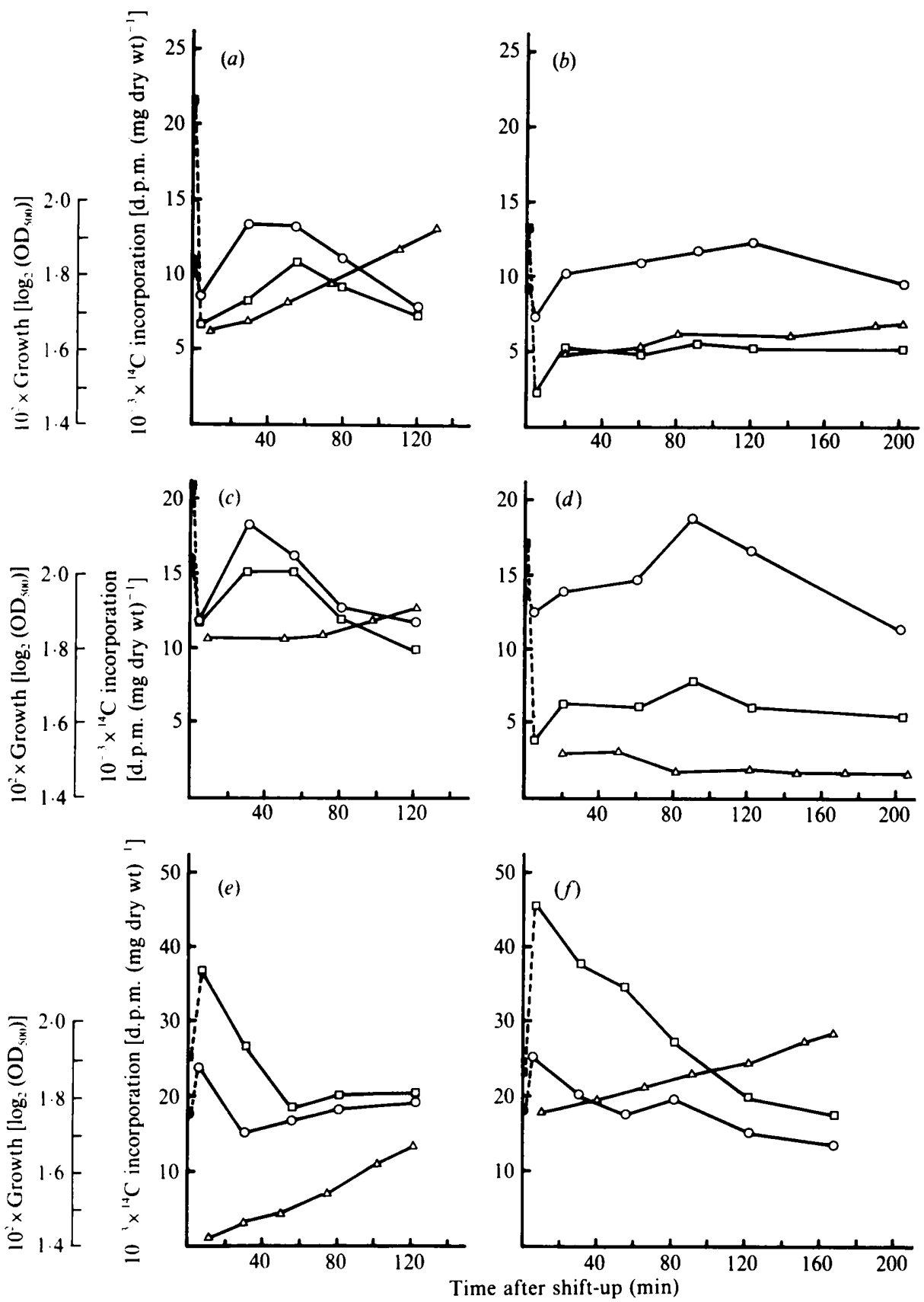

Fig. 5. Effect of shift-up in different solutes on growth and phospholipid synthesis in $\boldsymbol{V}$. costicola. Cultures growing in $1 \mathrm{M}-\mathrm{NaCl}$ were shifted-up in media containing final (equivalent) solute concentrations of $2 \mathrm{M}(a, c, e)$ or $3 \mathrm{M}(b, d, f), \mathrm{NaCl}(a, b)$ sucrose $(c, d)$ and glycerol $(e, f)$. Synthesis of phosphatidylglycerol $(O)$ and phosphatidylethanolamine $(\square)$ was measured as described in Methods. The growth of cultures was monitored by OD $_{500}$ measurements $(\triangle)$. 
The effect on growth of a shift-up in 2 and $3 \mathrm{M}$-sucrose media was more severe, compared with equivalent $\mathrm{NaCl}$ media (cf. Fig. $5 a, b, c, d$ ): the growth rate in $2 \mathrm{M}$-sucrose medium was slower, and the lag period longer, whilst there was no growth in $3 \mathrm{M}$-sucrose medium (Fig. $5 c, d$ ).

In contrast, as noted above for initial inhibition of phospholipid synthesis, the effects of shiftup in glycerol media on the time-course of phospholipid synthesis were quite different (Fig. $5 e, f)$. Instead of an initial inhibition of $\mathrm{PG}$ or PE synthesis, there was a stimulation which was greatest for PE. This initial increase in rates of phospholipid synthesis was followed by decline to near the control rates in $1 \mathrm{M}-\mathrm{NaCl}$. There was no increase in the rate of PG synthesis over that of PE synthesis, which remained higher throughout the time-course. The growth of cultures was much less affected by shifts-up in glycerol. Compared with $\mathrm{NaCl}$ or sucrose media there was no lag period, even when the shift-up was to $3 \mathrm{M}$-glycerol; the final growth rates in the $3 \mathrm{M}$-glycerol shifts-up were higher than those in $3 \mathrm{M}-\mathrm{NaCl}$ or sucrose media (Fig. 5), confirming the results shown in Fig. 3.

\section{DISCUSSION}

This work was undertaken to provide answers to two questions arising from our earlier work on haloadaptation in $V$. costicola. (1) What is sensed by the organism (i.e. is it the change in salt concentration or the absolute salt concentration in the medium)? (2) What part is played by osmotic effects in the response to high salinity? Thus, one of the aims of these experiments was to give an insight into the salt-sensing mechanism in moderately halophilic bacteria. In a recent review (Russell \& Kogut, 1985) we reasoned that the cell membrane or envelope may be important in sensing salt concentrations and in mediating the haloadaptive response. The absolute concentration of salt might be sensed as a graded effect on a sensitive enzyme(s), whereas the magnitude of a change could be monitored by, for example, an ion pump in the cytoplasmic membrane. Changes in phospholipid composition could play a part by altering the activity of such membrane-bound enzymes.

Our present results show that the magnitude of the change in salt concentration plays the predominant role in determining the final growth rate after a shift-up. This was exemplified particularly by the fact that the final growth rate in 2 and $3 \mathrm{M}-\mathrm{NaCl}$ was faster following a 2-fold shift-up than a 3-fold shift-up. The importance of the magnitude of the shift was also demonstrated by experiments in which cultures were shifted-up from $0.5 \mathrm{M}-\mathrm{NaCl}$ to $1.0 \mathrm{M}$ (a 2fold increase) or to $1.5 \mathrm{M}-\mathrm{NaCl}$ (a 3-fold increase). At the initial concentration of $0.5 \mathrm{M}-\mathrm{NaCl}$, growth was suboptimum, whereas at 1 and $1.5 \mathrm{M}-\mathrm{NaCl}$, growth rates were in the optimum range (cf. Fig. $3 a$ ). Therefore, a response to absolute $\mathrm{NaCl}$ concentration should have increased growth rates $(\mu)$ to the optimum (about $0.7-0.8$ ), whereas the actual response was a slight increase in growth rate (to 0.57 ) in the shift-up from 0.5 to $1.0 \mathrm{M}-\mathrm{NaCl}$, and a decline (to 0.41 ) in the shift-up from 0.5 to $1.5 \mathrm{M}-\mathrm{NaCl}$. The presence and length of lag periods in growth after shiftsup seem to be influenced both by the final salt concentration and the magnitude of the shift. It is difficult to assess which of these predominates. A shift-up to $3 \mathrm{M}-\mathrm{NaCl}$ always produced a considerable lag in growth, irrespective of the pre-shift salt concentration, and shifts-up to $1.5 \mathrm{M}$ $\mathrm{NaCl}$ never produced a lag. Shifts-up to $2 \mathrm{M}-\mathrm{NaCl}$ produced a lag when the change was 3-fold, but not when it was 2-fold.

Changes in membrane phospholipid synthesis appear to be part of the haloadaptive response in $V$. costicola (Kogut \& Russell, 1984; Russell et al., 1985). Because of the immediate onset and rapid reversibility of the initial inhibition in phospholipid synthetic rates after shift-up in $\mathrm{NaCl}$ (Russell et al., 1985) we thought that this could be due to a direct effect of $\mathrm{NaCl}$ (or one of its ions) on one or more phospholipid biosynthetic enzymes, located in the cytoplasmic membrane (Harwood \& Russell, 1984). From the data in Fig. 2, it is clear that the initial inhibition of phospholipid synthesis after a shift-up depended predominantly on the magnitude of the shift, rather than on the final $\mathrm{NaCl}$ concentration: thus, in cultures shifted-up from 0.7 and $1.0 \mathrm{M}$ $\mathrm{NaCl}, 50 \%$ initial inhibition occurred in approximately 1.6 and $2.5 \mathrm{M}-\mathrm{NaCl}$ respectively. This indicates that the initial inhibition of phospholipid synthesis is not due, simply, to the direct effect of salt concentration, i.e. on the activity or conformation of one or more enzymes. Thus, 
in this response, as in the response of growth rates to various shifts-up in external salt concentration, the bacteria appeared to retain a 'memory' of the original salt concentration, and this could persist for several hours. Such a 'memory' effect could be explained if, after a sudden change in external salinity, either the bulk internal salt concentration, or the $\mathrm{Na}^{+}$or $\mathrm{Cl}^{-}$ concentrations in the locality of enzyme(s) critical for growth and phospholipid synthesis, did not equilibrate rapidly after the shift. In other words, the magnitude of the shift could be related to osmotic stress imposed by sudden increase in external salt concentration. Although Schindler et al. (1977) reported that internal (cell-associated) salt concentrations in $V$. costicola grown isotonically at various salinities were approximately the same as those of the external media, much lower concentrations of free ions may exist intracellularly (Kushner, 1978; Y. Avidor, H. Gilboa \& M. Kogut, unpublished results). Ion gradients across the outer or plasma membrane could thus be part of the salt sensing/effector system (Russell \& Kogut, 1985).

This possibility and the role of osmotic effects in the adaptive responses have been investigated by examining the behaviour of $V$. costicola in media containing equivalent concentrations of sucrose - as a non-ionic, non-penetrant solute - and glycerol as a non-ionic solute that penetrates readily (M. Kogut, unpublished result). Examination of the growth rates over equivalent concentration ranges of the three solutes (Fig. 3) indicates that sucrose is less well tolerated than $\mathrm{NaCl}$, whereas glycerol shows a profile similar to that of $\mathrm{NaCl}$. The salt range of $\boldsymbol{V}$. costicola and the relationship between $\mathrm{NaCl}$ concentration and growth yield were known already (Forsyth \& Kushner, 1970). However, it was necessary to establish how growth rates changed with solute concentrations in order to establish baselines for the subsequent experiments in which solute concentrations were shifted. We also established that whilst there is a minimum concentration of $0.5 \mathrm{M}-\mathrm{NaCl}$ for growth of $V$. costicola in complex media, this is reduced to $0.3 \mathrm{M}-\mathrm{NaCl}$ when sucrose or glycerol are used to make the total concentration equivalent to 0.5 or $1.0 \mathrm{M}$ (Table 1). However, no growth occurred when sucrose or glycerol were used at equivalent concentrations of $1.0 \mathrm{M}$ in the absence of $\mathrm{NaCl}$. It appears, therefore, that there is a specific requirement for $\mathrm{NaCl}$ (or similar salts) of approximately $0.3 \mathrm{M}$ and a more general (osmotic?) solute requirement of about $0.5 \mathrm{M}$. When cells of $V$. costicola are suspended in media containing $0.3 \mathrm{M}$ total solute concentration they round up and often lyse, an observation which adds weight to the claim that this bacterium requires osmotic support.

Comparison of the effects of the three solutes on phospholipid synthesis (Figs 4 and 5) shows that, although the responses are broadly similar when the osmolarity of the culture medium is raised with sucrose instead of $\mathrm{NaCl}$, there are some differences. For example, the initial (i.e. 5 to 20 min after shift-up) rate of PE synthesis is inhibited less by sucrose at low concentrations than by equivalent concentrations of $\mathrm{NaCl}$ (Fig. 4), whilst the initial rates of PG synthesis appear even to be stimulated by $<2 \mathrm{M}$-sucrose. At the higher concentrations sucrose is more inhibitory for the initial rates of PE and (to a lesser extent) PG synthesis than are equivalent concentrations of $\mathrm{NaCl}$.

The data in Fig. 5, pertaining to the time-courses of growth and phospholipid synthesis rates after shifts-up to 2 and $3 \mathrm{M}$-sucrose and $\mathrm{NaCl}$, confirm that sucrose has a more severe effect on growth. There is a substantial lag after the shift-up to $2 \mathrm{M}$-sucrose, but not to $2 \mathrm{M}-\mathrm{NaCl}$, and the final growth rate is also lower in sucrose. A shift-up to $3 \mathrm{M}$-sucrose gave no recovery in growth after more than $4 \mathrm{~h}$. Shifts-up to $2.5 \mathrm{M}$-sucrose had intermediate effects (data not shown). On the other hand, the changes in phospholipid synthesis after these shifts-up in sucrose and $\mathrm{NaCl}$ concentrations are qualitatively and quantitatively similar. In particular, there is the same 'switch-over' in PG and PE synthesis rates following shifts-up in $\mathrm{NaCl}$ or sucrose media, which thus accounts for the increase in proportion of $P G$ relative to PE that occurs after shift-up in $\mathrm{NaCl}$ (Kogut \& Russell, 1984) or sucrose (data not shown) media. Thus, it appears that osmotic pressure, rather than a change in concentration of one or more types of ion, is most likely to be the trigger for this aspect of haloadaptation at least.

Cultures shifted-up to $3 \mathrm{M}$-sucrose media did not grow, but the same phospholipid synthesis changes occurred as on shift-up to $2 \mathrm{M}$-sucrose media when there was growth (Fig. 5). This confirms indications from earlier experiments that changes in phospholipid synthesis and composition do not depend on concomitant protein synthesis. 
The effects of shifts-up in glycerol media are in complete contrast to those in sucrose and $\mathrm{NaCl}$. The initial rates of $\mathrm{PE}$ and $\mathrm{PG}$ synthesis are stimulated by $₹ 2 \mathrm{M}$-glycerol concentrations, and PE synthesis is not inhibited even at $2.5 \mathrm{M}$-glycerol (Fig. 4). The time-courses of growth and phospholipid synthesis after shifts-up to 2 and $3 \mathrm{M}$-glycerol showed no lag, even at $3 \mathrm{M}$, and the growth rates were faster than after the shifts-up in equivalent $\mathrm{NaCl}$ concentrations. Finally, there was no 'switch-over' in the relative rates of PG and PE synthesis, even after a shift-up to $3 \mathrm{M}$-glycerol; the rate of PE synthesis remained above that of PG synthesis throughout.

In general, our main conclusion that osmotic factors must play a substantial role in the haloadaptation of $\boldsymbol{V}$. costicola gains support from our experiments with glycerol, which is known to be a penetrant solute for this organism. Thus phenotypic haloadaptation of this organism must involve sensing and response to osmotic pressure. This could be mediated by changes in lateral pressure in the cytoplasmic membrane, where the phospholipid synthesis enzymes are located, or by more specific local changes in membrane phospholipid conformation.

On the other hand, the irreplaceable minimum requirement of $\mathrm{NaCl}$ (or similar salts) for growth also point to a specific role for $\mathrm{NaCl}$ (or one of its ions). Macleod (1986) has identified an osmotic function of $\mathrm{NaCl}$ for the retention of accumulated solutes in the moderate halophile Alteromonas haloplanktis, and in V. costicola, although sucrose was not investigated. It seems likely that different cellular functions will respond differently to ionic and non-ionic solutes in various halophiles. In view of its complex behaviour towards ionic and non-ionic solutes, $V$. costicola could be termed an 'osmohalophile'.

This work was supported by project grants (to N.J.R.) from SERC and AFRC.

\section{REFERENCES}

FORSYTH, M. P. \& KuSHNER, D. J. (1970). Nutrition and distribution of salt response in populations of halophilic bacteria. Canadian Journal of Microbiology 16, 253-261.

hanna, K., Bengis-Garber, C., Kogut, M., KushNER, D. J. \& KaTES, M. (1984). The effect of salt concentration on the phospholipid and fatty acid composition of the moderate halophile Vibrio costicola. Canadian Journal of Microbiology 30, 669-675.

HaRwoOd, J. L. \& Russell, N. J. (1984). Lipids in Plants and Microbes, pp. 96-101. London: George Allen and Unwin.

KATES, M. (1972). Technigues of Lipidology: Isolation, Analysis and Identification of Lipids, pp. 349-352. Amsterdam: North-Holland Publishing Co.

KoGuT, M. \& Russell, N. J. (1984). The growth and phospholipid composition of a moderately halophilic bacterium during adaptation to changes in salinity. Current Microbiology 10, 95-98.

KUSHNER, D. J. (1978). Life in high salt and solute concentrations: halophilic bacteria. In Microbial Life in Extreme Environments, pp. 318-368. Edited by D. J. Kushner. London: Academic Press.

Kushner, D. J., Hamaide, F. \& Macleod, R. A. (1983). Development of salt-resistant active transport in a moderately halophilic bacterium. Journal of Bacteriology 153, 1163-1171.

Macleod, R. A. (1986). Salt requirements for membrane transport and solute retention in some moderate halophiles. FEMS Microbiology Reviews 39, 109113.
MilleR, K. J. (1985). Effects of temperature and sodium chloride concentration on the phospholipid and fatty acid compositions of a halotolerant Planococcus sp. Journal of Bacteriology 162, 263-270.

Russell, N. J. \& KoGUT, M. (1985). Haloadaptation: salt sensing and cell-envelope changes. Microbiological Sciences 2, 345-350.

Russell, N. J., Kogut, M. \& Kates, M. (1985). Phospholipid synthesis in the moderately halophilic bacterium Vibrio costicola during adaptation to changing salt concentrations. Journal of General Microbiology 131, 781-789.

Russell, N. J., Adams, R., Bygraves, J. \& Kogut, M. (1986). Cell envelope phospholipid changes in a moderate halophile during phenotypic adaptation to altered salinity and osmotic stress. FEMS Microbiology Reviews 39, 103-107.

SCHINDLER, D. B., WYDRo, R. M. \& KUSHNER, D. J. (1977). Cell-bound cations of the moderately halophilic bacterium Vibrio costicola. Journal of Bacteriology 130, 698-703.

SCHNAIDERMAN, R. \& Avi-DoR, Y. (1982). The uptake and extrusion of salts by the halotolerant bacterium, $\mathrm{Ba}_{1}$. Biochimica et biophysica acta 213, 177-185.

Wolf, A. V., Brown, M. G. \& Prentiss, P. G. (1981). Concentrative properties of aqueous solutions: conversion tables. In CRC Handbook of Chemistry and Physics, 60th edn, pp. D227-D276. Edited by R. C. Weast. Boca Raton: CRC Press. 